Among the sets of values which satisfy (8) there is one set which also makes the coefficient of $h^{4}$ in (6) vanish. It is easily verified that this set is

$$
\begin{aligned}
& r=\frac{(5)^{1 / 2}}{10}=0.2236, \\
& \theta=\frac{3-(5)^{1 / 2}}{6}=0.1273 .
\end{aligned}
$$

The truncation error of (1) with the particular values (9) is therefore $0\left(h^{6}\right)$. This should provide a superior recurrence formula for integrating $\psi_{x x}=\psi_{t}$. As usual with implicit formulas it will be necessary to invert but this can be done once and for all as soon as the number of spatial subdivisions has been chosen.

\title{
REFERENCES
}

1. E. Schmidt, Über die Andwendung der Differenzenrechnung auf technische Anheiz-und-Abkühungsprobleme, A. Föppl Festschrift, Springer, Berlin, 1924, pp. 179-189

2. J. Crank and P. Nicolson, A practical method for numerical evaluation of solutions of partial differential equations of heat-conduction type, Proc. Camb. Phil. Soc. 32, 50-67 (1947)

3. G. G. O'Brien, M. A. Hyman and S. Kaplan, A study of the numerical solution of partial differential equations, J. Math. Phys. 29, 223-251 (1951)

4. W. E. Milne, Numerical solution of differential equations, John Wiley, N. Y., 1953, p. 122

5. C. M. Fowler, Analysis of numerical solutions of transient heat-flow problems, Quart. Appl. Math. 3, 361-376 (1946)

6. S. H. Crandall, Implicit vs. explicit recurrence formulas for the linear diffusion equation. J. Assoc. Comput. Mach. 2, 42-49 (1955)

7. See, for example, F. B. Hildebrand, Methods of applied mathematics, Prentice-Hall, N. Y., 1952, p. 330

8. For six possible alternatives see P. H. Price and M. R. Slack, Stability and accuracy of numerical solutions of the heat flow equation, Brit. J. Appl. Phys. 3, 379-384 (1952)

\section{ON A METHOD OF GENERATING THREE-DIMENSIONAL POTENTIAL FLOWS FROM TWO-DIMENSIONAL ONES*}

\author{
By CHIA-SHUN YIH (Ioma Institute of Hydraulic Research)
}

In 1951, A. Wintner ${ }^{1}$ presented a method of deriving three-dimensional potential flows from two-dimensional ones. This method was found by Weingarten ${ }^{2}$ over sixty years ago and rediscovered by Levi-Civita ${ }^{3}$ a decade later. Whereas it is surprising that the flows thus derived should have been forgotten in the intervening years, it is indeed strange that another method for the same purpose, which is very straightforward and simple, never seems to have been mentioned. In the following, this new method, which differs essentially from the geometrical method of Weingarten, will be presented together with some examples illustrating its practical applications.

For irrotational flow, the curl of the velocity vector is zero, and the velocity com-

*Received Dec. 2, 1954.

${ }^{1} \mathrm{~A}$. Wintner, On a geometrical method of deriving three-dimensional harmonic flows from two-dimensional ones, Quart. Appl. Math. 9, 102-105 (1951).

2,3See Ref. 1. 
ponents $u, v$, and $w$ in the directions of $x, y$, and $z$ can be derived from a potential $\phi$ in the following way:

$$
u=-\phi_{x}, \quad v=-\phi_{\nu}, \quad w=-\phi_{z},
$$

in which the subscripts denote partial differentiation. From Eqs. (1) and the equation of continuity

$$
u_{x}+v_{y}+w_{z}=0
$$

it follows that the velocity potential satisfies the Laplace equation

$$
\phi_{x x}+\phi_{y y}+\phi_{z z}=0 \text {. }
$$

If one puts

$$
\phi=f(x, y)+g(y, z)+h(z, x)
$$

and demands that

$$
f_{x x}+f_{y y}=0, \quad g_{y y}+g_{z z}=0, \quad h_{z z}+h_{x x}=0,
$$

Eq. (3) is evidently satisfied. Hence the

Theorem: Harmonic functions of any two of the variables $x, y$, and $z$ can be superposed to form a harmonic function of all three variables; or, physically, two-dimensional potential flows parallel to mutually perpendicular planes can be superposed to form a three-dimensional potential flow.

As is well known, harmonic functions of two variables can be obtained from an analytic function of the corresponding complex variable. For instance, the real parts as well as the coefficients of the imaginary number in the analytic functions $F(\alpha), G(\beta)$, and $H(\gamma)$ in which

$$
\alpha=x+i y, \quad \beta=y+i z, \quad \gamma=z+i x
$$

are all harmonic functions, and any number of them can be used for superposition to form a velocity potential for three-dimensional flow. However, to conform with established usage, the real parts of $F(\alpha), G(\beta)$, and $H(\gamma)$ will be taken to be the functions $f, g$, and $h$ in Eq. (4).

A few examples will now be given. If

$$
F(\alpha)=\alpha+\frac{a^{2}}{\alpha}, \quad G(\beta)=0, \quad H(\gamma)=\frac{i a^{2}}{\gamma},
$$

superposition of the two-dimensional flows gives the flow across a solid body consisting of a hub and four spokes. The cross sections of these spokes are approximately circles of radius $a$ at large distances from the hub. The velocity at large distances from the solid body is uniform and in the $x$-direction. Of course, the terms $a^{2} / \alpha$ and $i a^{2} / \gamma$ can be replaced by suitable functions so that the cross section of the vertical spokes and that of the horizontal ones may have any desirable shapes at large distances from the hub, so long as the corresponding two-dimensional flows are known.

The flow obtained from

$$
F(\alpha)=\alpha+C \operatorname{coth} \frac{\pi \alpha}{b}, \quad G(\beta)=0, \quad H(\gamma)=-C^{\prime} \operatorname{coth} \frac{\pi \gamma i}{d},
$$


in which $C$ and $C^{\prime}$ are real and positive, is the flow across a rectangular grid. The cross sections of the bars far from the intersections are roughly circular, particularly if $b$ is large compared with $C$, and $d$ is large compared with $C^{\prime}$.

The flow corresponding to

$$
F(\alpha)=C \alpha^{2}, \quad G(\beta)=D \beta^{2}, \quad H(\gamma)=E \gamma^{2},
$$

is one for which the coordinate planes are not pierced by streamlines and are therefore "streamline surfaces" perpendicular to equi-potential surfaces.

Three-dimensional flows constructed by the present method belong to a special class, of which two-dimensional flows form a sub-class. Axisymmetric flows, however, do not belong to this class.

The reviewer of the original manuscript of this paper suggested that the expressions of the velocity components in terms of the complex conjugates of $f, g$, and $h$ be also given. If the latter are denoted by $f^{\prime}, g^{\prime}$, and $h^{\prime}$, then the Cauchy-Riemann equations are

$f_{x}=f_{\nu}^{\prime}, \quad f_{\nu}=-f_{x}^{\prime} ; \quad g_{\nu}=g_{z}^{\prime}, \quad g_{z}=-g_{y}^{\prime} ; \quad h_{z}=h_{x}^{\prime}, \quad h_{x}=-h_{z}^{\prime}$

and one has, from Eqs. (1), (4), and (6):

$$
u=h_{z}^{\prime}-f_{\nu}^{\prime}, \quad v=f_{x}^{\prime}-g_{z}^{\prime}, \quad w=g_{y}^{\prime}-h_{x}^{\prime} .
$$

In vector form, Eq. (6) can be written as

$$
\mathbf{V}=-\operatorname{curl} \mathbf{A}
$$

in which

$$
\mathbf{A}=\mathbf{i} g^{\prime}+\mathbf{j} h^{\prime}+\mathbf{k} f^{\prime}
$$

( $\mathbf{i}, \mathbf{j}$, and $\mathbf{k}$ being unit vectors along the coordinate axes) is the vector potential for the flow for which $\phi$ is the scalar potential.

It may be remarked without further discussion that two-dimensional potential flows which are not parallel to mutually perpendicular planes can also be superimposed to form a three-dimensional potential flow.

\section{A NOTE ON THE MEAN VALUE OF RANDOM DETERMINANTS* BY RICHARD BELLMAN (The Rand Corporation)}

1. Introduction. In a recent paper, [1], Nyquist, Rice and Riordan discussed the problem of determining the expected values of powers of a random determinant. Here a random determinant, $D_{n}$, is defined to be

$$
D_{n}=\left|x_{i i}\right|, \quad i, j,=1,2, \cdots, n,
$$

where the $x_{i i}$ are independent random variables.

The purpose of the present note is to give an explicit representation for $E\left(D_{n}^{k}\right)$ in terms of the characteristic functions of the $x_{i i}$. These need not be identical.

At the moment we are merely interested in presenting an expression which will

*Received Dec. 14, 1954. 